\title{
Focusing light in scattering media by ultrasonically encoded wavefront shaping (SEWS)
}

Jian Wei Tay, Puxiang Lai, Yuta Suzuki, Lihong V. Wang

Jian Wei Tay, Puxiang Lai, Yuta Suzuki, Lihong V. Wang, "Focusing light in scattering media by ultrasonically encoded wavefront shaping (SEWS)," Proc. SPIE 8943, Photons Plus Ultrasound: Imaging and Sensing 2014, 89434P (3 March 2014); doi: 10.1117/12.2037037

SPIE. Event: SPIE BiOS, 2014, San Francisco, California, United States 


\title{
Focusing light in scattering media by ultrasonically-encoded wavefront shaping (SEWS)
}

\author{
Jian Wei Tay, Puxiang Lai, Yuta Suzuki, Lihong V. Wang
}

Optical Imaging Laboratory, Washington University in St Louis, One Brookings Drive, St Louis, MO 63101

\begin{abstract}
Wavefront distortion in scattering media can be compensated for using optical wavefront shaping. In this technique, a spatial light modulator (SLM) is used to apply a spatially distributed phase shift to the optical field. A genetic optimization algorithm was used to obtain the SLM pattern which best focuses light within the medium. The target volume is defined by using a focused ultrasound beam to encode light travelling within the acoustic focus. The ultrasonically-encoded light is measured and used as feedback to the algorithm, which then searches for the pattern which maximizes the encoded light intensity. We call this technique ultrasonically-encoded wavefront shaping (SEWS). Using SEWS, we focused light into a scattering medium consisting of ground glass diffuser and a gelatin phantom. The optical intensity at the target was increased by 11 times over the original intensity. These results were validated using fluorescent imaging at the ultrasonic focus.
\end{abstract}

Keywords: Optical Engineering, Wavefront Shaping, Turbidity Compensation, Ultrasound, Imaging, Spatial Light Modulator

\section{INTRODUCTION}

Being able to focus light into tissue is important for many biomedical optics applications. For example, in imaging applications, the optical focal spot determines the resolution, while for therapeutic and photo-manipulation applications, a focused beam both spatially defines the target region, as well as increases the available optical intensity in the region. However, focusing light in biological tissue, which fall under the category of scattering media, is challenging because refractive index inhomogeneities scramble the optical path length within the medium. Therefore, focusing light using conventional optical lenses is impossible beyond a single transport mean free path, which is on the order of a few millimeters in soft tissue [1]. The effect of scattering can be observed by illuminating the sample with a coherent source. The light exiting the sample self-interferes and forms a speckled intensity pattern.

(a)
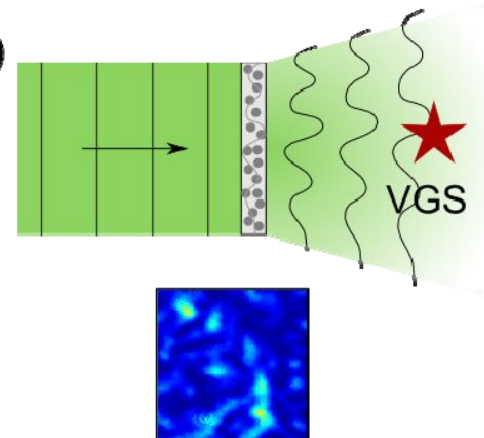

(b)

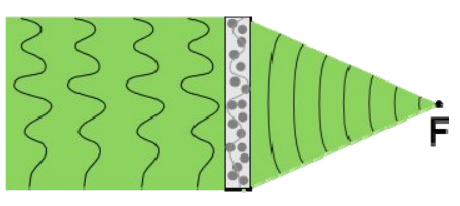

Figure 1: Illustration of optical wavefront engineering. (a) A scattering medium, such as ground glass, scrambles the optical field, resulting in a speckle pattern. (b) An optical focal spot can be formed by applying a spatially distributed phase shift to the illuminating light, compensating for scattering.

Photons Plus Ultrasound: Imaging and Sensing 2014, edited by Alexander A. Oraevsky, Lihong V. Wang, Proc. of SPIE Vol. 8943, 89434P · C 2014 SPIE · CCC code: 1605-7422/14/\$18 · doi: 10.1117/12.2037037 
Until recently, focusing through a scattering medium was thought to be impossible. However, advances in optical wavefront engineering have shown that this is not the case [2]. Scattering can be compensated for by using spatial light modulators (SLMs), which are essentially programmable phase cell arrays, to apply a spatially distributed phase shift to the input beam, thereby focusing light after the scattering medium. An illustration of this concept is shown in Fig. 1. The optimized SLM phase pattern which best focuses light to the target spot can be obtained by many different methods, for example by using iterative search algorithms $[3,4]$, or by directly measuring the phase distortion for each SLM element and applying the inverse [5].

Optical wavefront engineering has mostly been used to focus light through scattering media. Here, we extend the use of this technique to focus light into a scattering sample, which is more biologically relevant, using ultrasonically-encoded wavefront shaping (SEWS). To focus light into a scattering sample, the target volume must first be defined. We do so using a focused ultrasound beam to phase-modulate, or encode, the scattered light propagating within the medium. We then use a genetic search algorithm to determine the SLM pattern which maximizes the intensity of the ultrasonically-encoded light. In this aspect, the focused ultrasound beam acts as a "guide star"; by maximizing the ultrasonically-encoded light, an optical focus is formed at the ultrasound focal volume. Other guide stars could be used, such as embedded fluorescent particles [6] or even photoacoustics [7,8]. Compared to embedded particles however, using an ultrasonic beam is noninvasive, non-toxic and allows the position of the optical focus to be changed at will by simply translating the ultrasound transducer.

SEWS has the potential to impact a variety of optical techniques. For example, many current techniques, such as the optogenetic stimulation of neurons [9], require optical fibers to be embedded within the tissue to sufficiently deliver light to the target region. Thus, by focusing light into tissue, SEWS has the potential to enhance these techniques by removing the necessity of invasive procedures. SEWS would also enable optical imaging at depths beyond one transport mean free path. Compared to other imaging techniques such as X-ray, optical imaging is non-ionizing and nonharmful, while providing a controllable absorption contrast depending on the wavelength used.

\section{OPTICAL SETUP}

A simplified schematic of our experiment is shown in Figure 2. A computer-controlled SLM (Holoeye PLUTO) was used to engineer the beam illuminating the sample. The SLM response was calibrated to provide a $2 \pi$ phase shift linearly over 191 grayscale values [10].

For this proof-of-principle demonstration, we used a pair of ground glass diffusers as our sample to simulate a scattering medium. A clear gelatin layer was inserted between the diffusers to enable acoustic coupling. The ultrasound then propagated and modulated the scattered optical field after the first diffuser. We used five cycles of ultrasound at $6 \mathrm{MHz}$. The transducer used had a transverse focal width of $400 \mu \mathrm{m}$. To visualize the optical focus, we also inserted a gelatin bar containing fluorescent quantum dots in the ultrasound focal volume. A CCD camera was used to acquire images from above the sample.

An optical fiber bundle after the second diffuser collected the ultrasonically-encoded light and directed it to a photorefractive interferometer [11]. The interferometer was formed with a BSO crystal, with the reference beam at the same frequency as the unmodulated light. The photorefractive interferometer works as follows: First, a refractive index hologram was formed within the crystal, 
which recorded the complex amplitude of the interference field between the umodulated scattered field exiting the sample, and the reference beam. The crystal then behaved like an adaptive beamsplitter, and a part of the reference beam was diffracted into the same mode and direction as the signal beam. Thus, at the output of the interferometer, the transmitted signal beam and the diffracted reference beam added coherently, giving a signal that was proportional to the intensity of the scattered signal beam.

The photorefractive hologram is able to adapt only to fluctuations that are slower than the photorefractive response time of the crystal (measured to be about $100 \mathrm{~ms}$ ). Since the time-of-flight of the ultrasound pulse $(1.9 \mu \mathrm{s})$ was much shorter than the photorefractive response time, the photorefractive grating did not adapt to the modulated light, so the signal and diffracted reference beams were no longer in phase. The result was a reduction of the detected signal beam intensity after the crystal that was proportional to the intensity of ultrasonically-encoded light. This signal, detected by a photodiode, is subsequently fed back to the optimization algorithm.

We used a genetic algorithm to obtain the SLM pattern which maximized the ultrasonicallyencoded light intensity. By doing so, it follows that light was focused back to the ultrasound focus. The algorithm used was based on that described by Conkey et al. [4]. The SLM was divided into $20 \times 20$ independently controlled segments. We used a population size of 30 , with an initial mutation rate of 0.12 , and a final mutation rate of 0.01 .

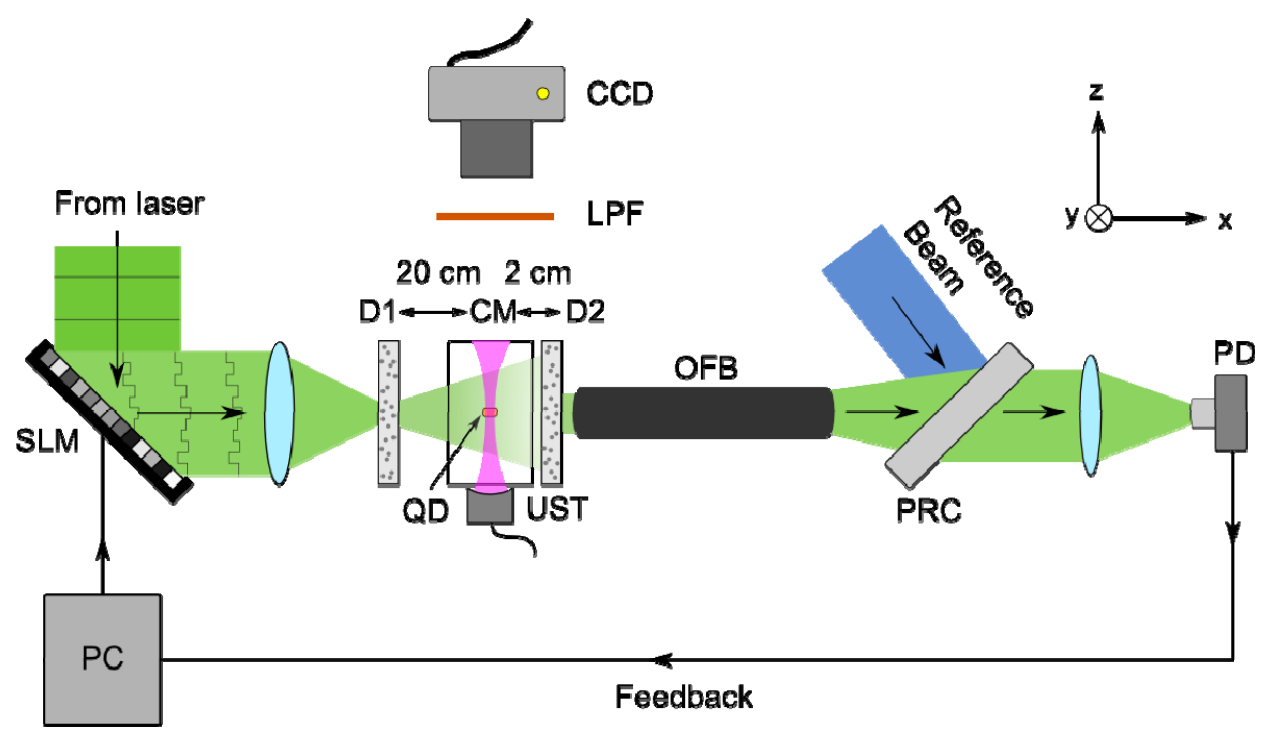

Figure 2: Schematic of the optical setup for SEWS. Abbreviations are CM: Clear gelatin medium; D1 \& D2: ground-glass diffusers; LPF: Low-pass filter; OFB: Optical fiber bundle; PD: Photodiode; PRC: photorefractive crystal; QD: Quantum dot bar; SLM: Spatial light modulator; UST: $6 \mathrm{MHz}$ Ultrasound transducer. 


\section{OPTIMIZATION RESULTS}
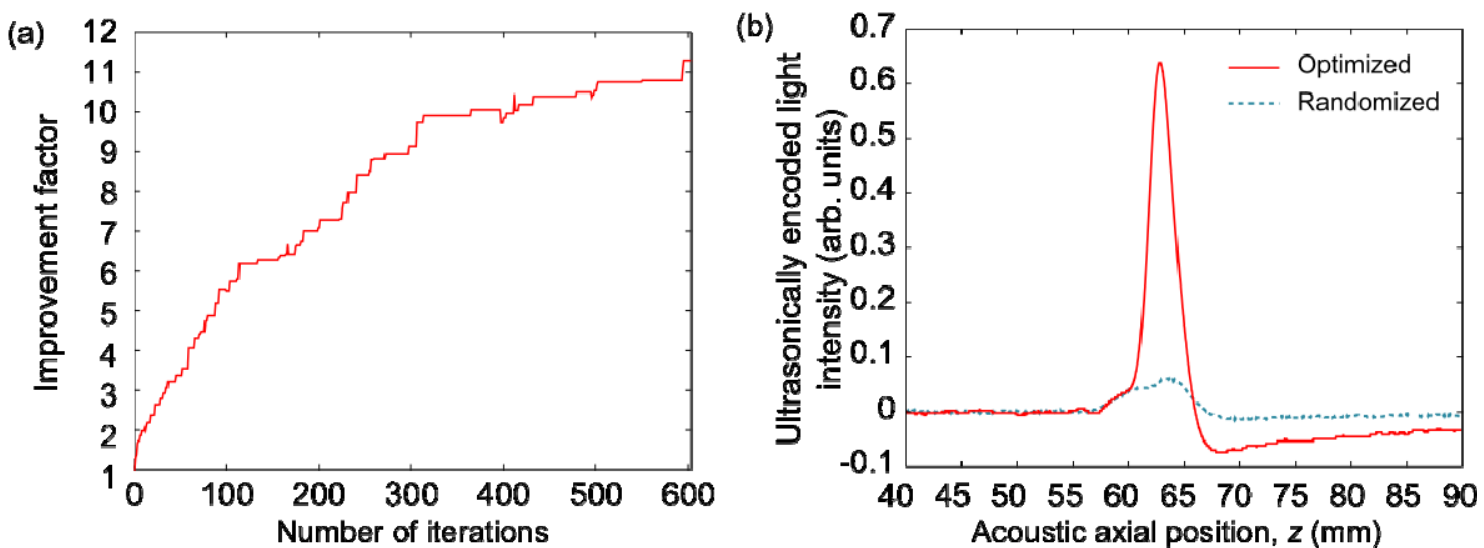

Figure 3: SEWS optimization results. (a) The increase of intensity of the ultrasonically-encoded light as the algorithm runs. (b) The ultrasonically-encoded light intensity, measured after the photorefractive interferometer, before (randomized) and after optimization. The results shown in (b) were averaged over 75 acquisitions.

After running the genetic algorithm for 600 iterations, the ultrasonically-encoded light intensity was increased by 11 times, as shown in Fig. 3. We also used a CCD camera above the sample, as shown in Fig. 2, to record the fluorescence intensity of the embedded bar, before and after the optimization, as shown in Fig. 4. The fluorescence intensity after optimization was increased by ten-fold, which agrees with the increase in the encoded light intensity. Note that the average fluorescence intensities recorded with a uniform pattern and a randomly generated pattern displayed on the SLM are similar, which indicates that the light is fully scrambled by the first diffuser.

\section{DISCUSSION}

From the optimization process, we obtained an increase in intensity of 11 times. The expected intensity increase has been shown [12] to follow the equation given by

$$
\varepsilon=\frac{\pi}{4} \frac{N-1}{M}
$$

where $N$ is the number of independently controlled segments on the SLM and $M$ is the number of optical speckles within the ultrasound focal zone. In our experiment, we divided the SLM into 20x20 segments, hence $N=400 . M$ was estimated from the size of a single optical speckle at the ultrasound position, which was estimated to be $130 \mathrm{um}$, and the FWHM of the transducer, which was measured to be $400 \mathrm{um}$. In our experiment, $M=22$. This gives us an expected intensity increase of $12 x$. Our experimental result therefore agrees well with the theoretical enhancement. 
(a)

(b)

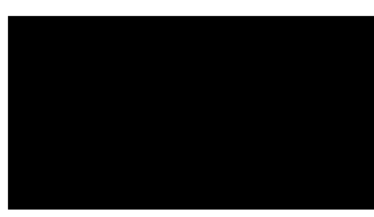

(c)
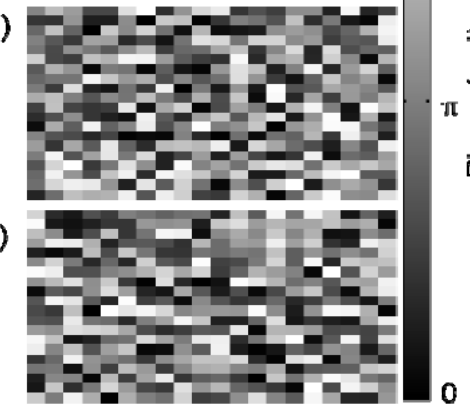

(d)

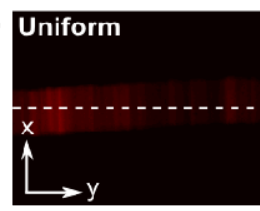

(e)

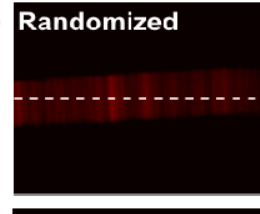

(f)

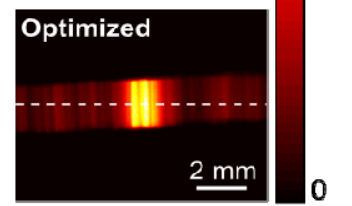

255

128

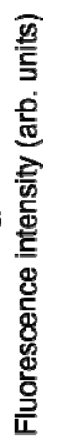

(g)

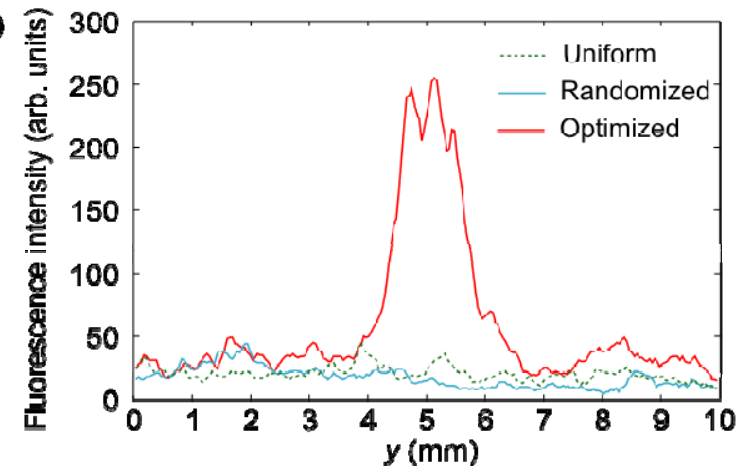

Figure 4: Fluorescence images from the quantum dot bar. (a) Uniform, (b) randomized, and (c) the optimized pattern displayed on the SLM The resulting CCD images of the flourescent bar are shown in (d)-(f). (g) The cross-sectional intensity, plotted along the white dotted lines in (d)-(f).

\section{CONCLUSION}

We have demonstrated SEWS, a method of focusing light into scattering media using ultrasonicallyencoded light as a guide star. By using ultrasonically-encoded light as feedback to a genetic optimization algorithm, a phase map was found which increased the intensity at the ultrasonic focal volume by an order of magnitude. Using ultrasound as a guide star is non-invasive, non-ionizing and non-harmful. We anticipate that SEWS will be useful for enhancing a variety of biomedical optics techniques, such as optogenetic stimulation of neurons, laser microsurgery, or optical imaging.

\section{ACKNOWLEDGEMENTS}

This work was sponsored in part by the National Academies Keck Futures Initiative grant IS 13 and National Institute of Health grant DP1 EB016986 (NIH Director's Pioneer Award). 


\section{REFERENCES}

[1] Wang, L. V. and Wu, H.-I., [Biomedical Optics: Principles and Imaging] (Wiley-Interscience, New Jersey, 2011).

[2] Vellekoop, I. M. and Mosk, A. P. "Focusing coherent light through opaque strongly scattering media," Opt. Lett. 32, 2309-2311 (2007).

[3] Vellekoop, I. M. and Mosk, A. P.. "Phase control algorithms for focusing light through turbid media," Optics Comm., 281, 3071-3080 (2008).

[4] Conkey, D. B., Brown, A. N., Caravaca-Aguirre, A. M. and Piestun, R.. "Genetic algorithm optimization for focusing through turbid media in noisy environments", Opt. Express 20, 4840-4849 (2012).

[5] Popoff, S. M., Lerosey, G., Carminati, R., Fink, M., Boccara, A. C. and Gigan, S.. "Measuring the transmission matrix in optics: an approach to the study and control of light propagation in disordered media," Phys. Rev. Lett. 104, 100601 (2010).

[6] Aulbach, J., Gjonaj, B., Johnson, P. and Lagendijk, A.. "Spatiotemporal focusing in opaque scattering media by wave front shaping with nonlinear feedback," Opt. Express 20, 29237-29251 (2012).

[7] Kong, F., Silverman, R. H., Liu, L., Chitnis, P. V., Lee, K. K. and Chen, Y. C.. "Photoacoustic-guided convergence of light through optically diffusive media," Opt. Lett. 36, 2053-2055 (2011).

[8] Chaigne, T., Katz, O., Boccara, A. C., Fink, M., Bossy, E. and Gigan, S. "Controlling light in scattering media noninvasively using the photoacoustic transmission matrix," N. Photon 8, 58-64 (2013).

[9] Fenno, L., Yizhar, O. and Deisseroth, K.. "The development and application of optogenetics," Annu. Rev. Neurosci. 34, 389-412. (2011).

[10] Tay, J. W., Taylor, M. A. and Bowen, W. P. "Sagnac-interferometer based characterization of spatial light modulators," Appl. Opt. 48, 2236-2242 (2009).

[11] Murray, T. W., Sui, L., Maguluri, G., Roy, R. A., Nieva, A., Blonigen, F. and DiMarzio, C. A. "Detection of ultrasound-modulated photons in diffuse media using the photorefractive effect." Opt. Lett. 29, 2509-2511 (2004).

[12] Vellekoop, I. M., van Putten, E. G., Lagendijk, a. \& Mosk, A. P., Demixing light paths inside disordered metamaterials. Opt. Express 16 (1), 67-80 (2008). 